\title{
Low-mass eclipsing binaries in the WFCAM Transit Survey
}

\author{
Patricia Cruz ${ }^{1}$, Marcos Diaz ${ }^{1}$, David Barrado ${ }^{2}$ and Jayne Birkby ${ }^{3,4}$ \\ ${ }^{1}$ Instituto de Astronomia, Geofísica e Ciências Atmosféricas, Universidade de São Paulo \\ (IAG/USP), Brazil \\ email: patricia.cruz@usp.br \\ ${ }^{2}$ Departamento de Astrofísica, Centro de Astrobiología (CAB/INTA-CSIC), Spain \\ ${ }^{3}$ Harvard-Smithsonian Center for Astrophysics, USA \\ ${ }^{4}$ NASA Sagan Fellow
}

\begin{abstract}
The characterization of short-period detached low-mass binaries, by the determination of their physical and orbital parameters, reveal the most precise basic parameters of lowmass stars. Particularly, when photometric and spectroscopic data of eclipsing binaries (EBs) are combined. Recently, 16 new low-mass EBs were discovered by the WFCAM Transit Survey (WTS), however, only three of them were fully characterized. Therefore, new spectroscopic data were already acquired with the objective to characterize five new detached low-mass EBs discovered in the WTS, with short periods between 0.59 and 1.72 days. A preliminary analysis of the radial velocity and light curves was performed, where we have derived orbital separations of 2.88 to $6.69 R_{\odot}$, and considering both components, we have found stellar radii ranging from 0.40 to $0.80 R_{\odot}$, and masses between 0.24 and $0.71 M_{\odot}$. In addition to the determination of the orbital parameters of these systems, the relation between mass, radius and orbital period of these objects can be investigated in order to study the mass-radius relationship and the radius anomaly in the low main-sequence.
\end{abstract}

Keywords. Eclipsing binaries, low-mass stars, spectroscopy

\section{Introduction}

Combined photometric and spectroscopic phase-resolved data of eclipsing binaries (EBs), and particularly double-lined EBs, provide the most precise ways to measure their fundamental properties without using spectral models.

Presently, only a small number of low-mass eclipsing binaries (LMEBs) was characterized with a good precision. As few as $\sim 20$ low-mass systems have stellar masses and radius measured with uncertainties below $5 \%$. Surprisingly, the data present in the literature when compared to stellar models present significant discrepancy, specially concerning the stellar radius. The M-dwarf stars in LMEBs seem to have radii 5 to $10 \%$ greater than the expected radius, derived from spectral models (López-Morales \& Ribas 2005, Kraus et al. 2011). This is known as the radius anomaly and it is a recurring problem of the determination of physical parameters of low-mass stars.

The discovery and characterization of new LMEBs will help unveiling the radius anomaly problem and set new limits on the mass-radius relationship of low-mass stars.

\section{Data analysis}

The WFCAM Transit Survey. The WFCAM Transit Survey (WTS) was focused on the discovery and study of exoplanets around low-mass stars. This survey has been awarded 200 nights with the Wide Field Camera (WFCAM) on the 4m UK Infrared 
Table 1. System parameters: preliminary results.

\begin{tabular}{lccccc}
\hline & $17 \mathrm{e}-3-02003$ & $17 \mathrm{~h}-4-01429$ & $19 \mathrm{c}-3-08647$ & $19 \mathrm{f}-4-05194$ & $19 \mathrm{~g}-2-08064$ \\
\hline $\mathrm{P}_{\text {orb }}($ days $)$ & 1.2250074 & 1.4445895 & 0.8674656 & 0.5895297 & 1.7204092 \\
error & 0.0000005 & 0.0000003 & 0.0000001 & 0.0000001 & 0.0000004 \\
\hline $\mathrm{M}_{1}\left(M_{\odot}\right)$ & 0.595 & 0.503 & 0.392 & 0.536 & 0.714 \\
error & 0.021 & 0.016 & 0.018 & 0.015 & 0.027 \\
\hline $\mathrm{M}_{2}\left(M_{\odot}\right)$ & 0.508 & 0.409 & 0.243 & 0.388 & 0.642 \\
error & 0.017 & 0.012 & 0.013 & 0.011 & 0.026 \\
\hline $\mathrm{R}_{1}\left(R_{\odot}\right)$ & 0.643 & 0.510 & 0.499 & 0.608 & 0.796 \\
error & 0.017 & 0.005 & 0.017 & 0.009 & 0.010 \\
\hline $\mathrm{R}_{2}\left(R_{\odot}\right)$ & 0.445 & 0.418 & 0.401 & 0.487 & 0.465 \\
error & 0.023 & 0.005 & 0.021 & 0.008 & 0.007 \\
\hline $\log \mathrm{g}_{1}$ & 4.596 & 4.724 & 4.635 & 4.599 & 4.490 \\
error & 0.022 & 0.007 & 0.029 & 0.011 & 0.008 \\
\hline log $\mathrm{g}_{2}$ & 4.847 & 4.808 & 4.616 & 4.652 & 4.910 \\
error & 0.045 & 0.009 & 0.045 & 0.015 & 0.012 \\
\hline $\mathrm{a}_{\text {orb }}\left(R_{\odot}\right)$ & 4.976 & 5.213 & 3.288 & 2.881 & 6.686 \\
error & 0.054 & 0.052 & 0.052 & 0.026 & 0.083 \\
\hline incl $\left.{ }^{\circ}\right)$ & 82.43 & 89.12 & 81.47 & 83.46 & 83.91 \\
error & 0.19 & 0.14 & 0.14 & 0.18 & 0.05 \\
\hline $\mathrm{T}_{\text {eff }, \mathrm{SED}}(\mathrm{K})$ & 3500 & 3400 & 3800 & 4200 & 4200 \\
\hline $\mathrm{T}_{\text {eff }, 1}(\mathrm{~K})$ & 3800 & 3400 & 3900 & 4400 & 4200 \\
\hline $\mathrm{T}_{\text {eff }, 2}(\mathrm{~K})$ & 3100 & 3100 & 3100 & 3500 & 3200 \\
\hline \hline
\end{tabular}

Telescope (UKIRT) to search for planets via the transit method at infrared wavelengths. The observations were performed in the WFCAM J band $\left(\lambda_{c}\right.$ at $\left.1.25 \mu \mathrm{m}\right)$, near to the maximum of the spectral energy distribution (SED) of low-mass stars. As a secondary objective, the survey also detected several light curves of eclipsing binaries (EBs). For instance, a fine number of light curves of low-mass EBs was discovered with short periods of less than 5 days (Birkby et al. 2012). He have then selected light curves of five new detached low-mass EBs discovered in the WTS, with short periods between 0.59 and 1.72 days.

SED fitting. We performed the SED fitting using the Virtual Observatory tool VOSA (Bayo et al. 2008). The filters considered were: SDSS u, g, r, i, z; WFCAM Z, Y, J, H, K; 2MASS J, H, Ks; WISE W1, W2. These data were available in the literature and allowed estimates of the combined effective temperatures of the EBs, which are shown in table 1.

Low-resolution spectroscopy. Low-resolution spectroscopic data were acquired with the TWIN spectrograph mounted on the 3.5m-telescope at the Calar Alto Observatory (CAHA) to derive the effective temperature of each component of the binary. We comparared the observed spectra with the BT-Settl library of synthetic spectra from Allard et al. (2013), by combining two synthetic spectra in order to reproduce the flux-calibrated observed spectra. The obtained individual temperatures $\left(\mathrm{T}_{\mathrm{eff}, 1}, \mathrm{~T}_{\mathrm{eff}, 2}\right)$ are also shown in table 1.

Radial velocity measurements. We have gathered intermediate resolution spectra also with TWIN/3.5m-telescope (CAHA) to measure radial velocity shifts (RVs) of the 5 

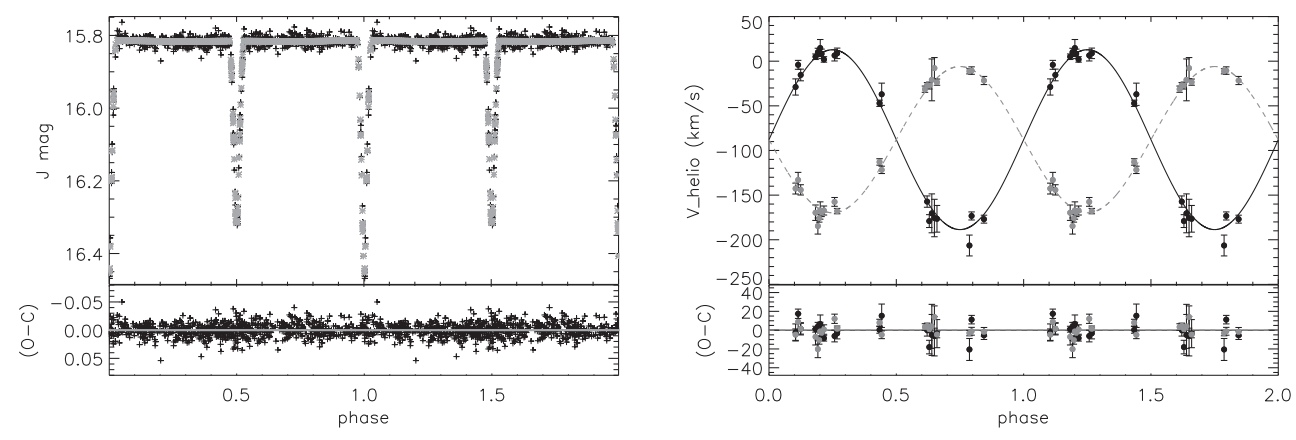

Figure 1. Light curve and RV fittings for the LMEB 17h-4-01429.

double-lined LMEBs. The RVs were computed via the Fourier cross-correlation of $\mathrm{H} \alpha$ emission components using IRAF's FXCOR.

Solving the systems. We have used the jktEBOP code (Southworth et al. 2004a,b) to analyze all photometric and spectroscopic data together and obtain an unique solution for each EB system. The orbital and physical parameters obtained from jktEBOP, with respective errors, are presented in table 1 . Figure 1 shows, as an example, the best fitting solution for the RV data and light curve of the LMEB 17h-4-01429.

\section{Discussion}

We have presented the current status of our project on the study of low-mass eclipsing binaries. The complete characterization of five new detached low-mass EBs discovered in the WTS is only possible by combining sets of photometric and spectroscopic data. There are few high precision determination of masses and radii for low-mass stars in shortperiod binaries. Between the analyzed systems, 3 EBs have M-dwarf stars as primary and secondary components, and 2 EBs with M-dwarfs as secondaries, with late K-dwarfs as primaries. This is an ongoing work, and we have presented preliminary results for all 5 systems. Future analysis steps include placing these objects in the mass-radius diagram and investigating the radius anomaly in low-mass binaries and its relation with their basic properties.

\section{References}

Allard, F., Homeier, D., Freytag, B., Schaffenberger, W., \& Rajpurohit, A. S. 2013, MSAIS, 24, 128

Bayo, A., Rodrigo, C., Barrado, Y., Navascués, D., Solano, E., Gutiérrez, R., Morales-Calderón, M., \& Allard, F. 2008, A\&̊A, 492, 277

Birkby, J., Nefs, B., Hodgkin, S., Kovács, G., Sipőcz, B., Pinfield, D., Snellen, I., Mislis, D., Murgas, F., Lodieu, N., de Mooij, E., Goulding, N., Cruz, P., Stoev, H., Cappetta, M., Palle, E., Barrado, D., Saglia, R., Martin, E., \& Pavlenko, Y. 2012, MNRAS, 426, 1507

Kraus, A. L., Tucker, R. A., Thompson, M. I., Craine, E. R., \& Hillenbrand, L. A. 2011, ApJ, 728,48

López-Morales, M. \& Ribas, I. 2005, ApJ, 631, 1120

Southworth, J., Maxted, P. F. L., \& Smalley, B. 2004a, MNRAS, 351, 1277

Southworth, J., Zucker, S., Maxted, P. F. L., \& Smalley, B. 2004b, MNRAS, 355, 986 